\title{
Teaching the Importance of Data Correlation in Engineering Technology
}

\author{
Michael R. Warren, Dana M. Burnett, Jay R. Porter, and Rainer J. Fink \\ Texas A\&M University
}

\begin{abstract}
To meet the needs of the semiconductor test industry, entry-level test engineers must understand the importance of correlation between characterization test equipment and industrial automated test platforms (ATEs). The expectation is that the test engineer should be able to use data from multiple platforms to aid in debugging device designs and test programs. As part of the Semiconductor Testing Initiative, the Electronics Engineering Technology Program at Texas A\&M University has begun to address this issue in their mixed-signal test course sequence.
\end{abstract}

Using a DAC0808 digital-to-analog converter as a test chip, students correlate the data between a Teradyne A567 tester and a National Instruments', Inc. test system with LabVIEW 6i and a PCI6025E data acquisition card. Several test-related issues are then explored including debugging, limitations of test equipment, and device interface board design constraints.

\section{Introduction}

The Electronics Engineering Technology (EET) Program at Texas A\&M University in conjunction with Texas Instruments has formed a Semiconductor Test Initiative to help find new and dynamic methods of teaching concepts in mixed signal test. [1] This initiative began with the donation of a Teradyne A567 automated production (ATE) tester by Texas Instruments, Inc. and Teradyne, Inc., which is currently being used in the advanced mixed signal test course. This is one of two mixed signal test courses that are currently offered within the EET program. The introductory and advanced mixed signal courses give students a hands-on experience in the field of mixed signal test. By using both the ATE tester and a PC-based lab station, a variety of topics in mixed signal test taught in the classroom can be reinforced through a laboratory experience. One topic that is of particular interest is that of data correlation between test platforms.

Data correlation refers to the ability to test a device on different hardware or software platforms and still obtain the same results. Correlation between different platforms is a problem that mixed-signal test and design engineers continually face during a product's life cycle. [2] For example, a design engineer evaluating performance might test a new device in a characterization lab. At the same time, a test engineer preparing for production is also testing the device on an ATE. Quite often, similar tests will 
yield different results on the two platforms. Correlation of these tests will often uncover problems in the design and/or test architectures.

To help future engineers understand the problems associated with correlation between platforms, the Advanced Mixed-Signal Test course exposes students to the process of data correlation in a laboratory setting. Using both a PC-based tester and an ATE tester, student teams test the linearity performance of a digital-to-analog converter (DAC). Once the tests are complete, the students use their data to compare results obtained on the different platforms and between different teams. This process teaches the students how to use test correlation to debug hardware and software and how to evaluate the performance of different test equipment.

\section{The Two Test Platforms}

Figure 1 shows the two testing platforms. The Teradyne A567 ATE, donated by Texas Instruments and Teradyne, can be seen on the right. It is a production grade semiconductor tester that allows for precision voltage and current measurements and the generation and capture of static or dynamic digital signals of up to 16-bit patterns. To support devices which use AC signals, the tester also sources and captures precision low-frequency waveforms. By necessity, production grade testers are built with the ability to rapidly test large volumes of devices, which is a major contributor to their high cost.

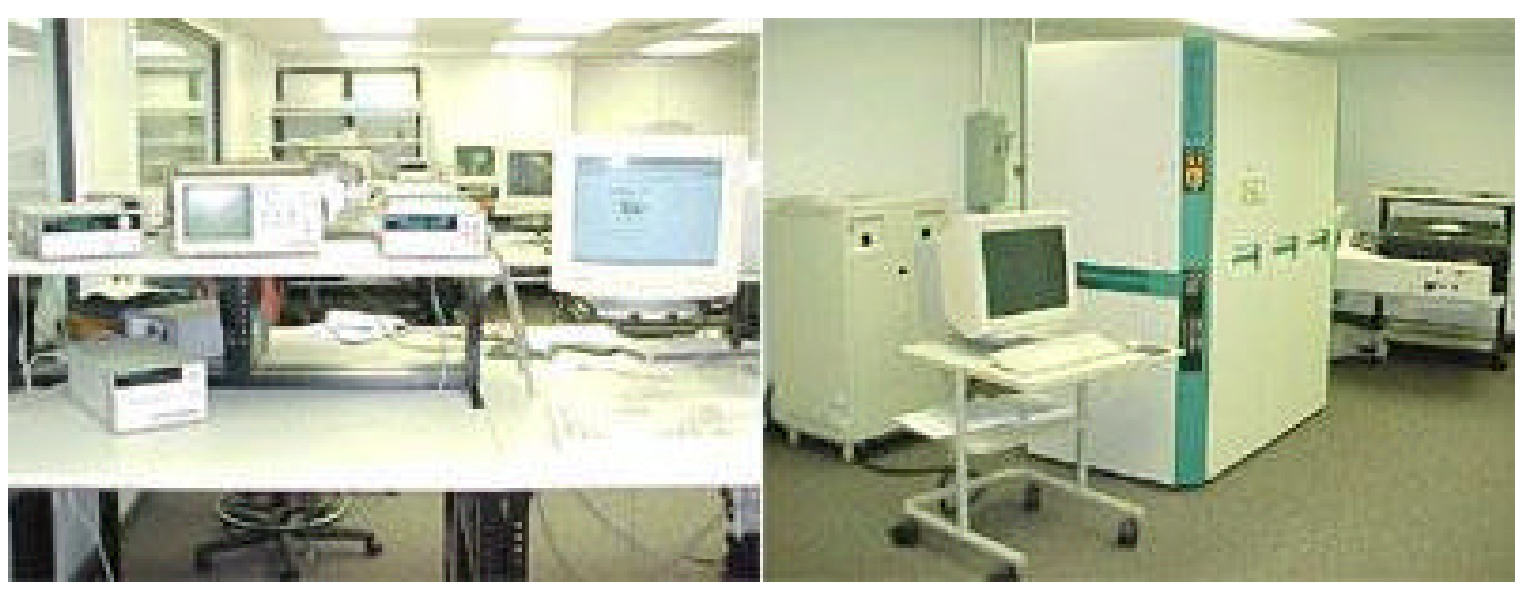

Figure 1 - PC-Based test setup (left) and a Teradyne A567 (right).

The present configuration of the A567 consists of one workstation, one test head, and the test equipment mainframe. The programming software on the A567 is Teradyne's IMAGE software package. Designed specifically for ATE's, IMAGE is a programming editor and compiler similar to C that allows for the automation of test equipment in the A567. In addition to the tester, sixteen device interface boards (DIBs) with protoboard interfaces have been donated to allow students to quickly build circuits to be tested on the ATE. 
The PC-based characterization platform can be seen on the left in Figure 1. Currently, the analog electronics laboratory is equipped with eleven of these stations. This allows introductory students to become familiar with mixed signal test concepts without having to tackle the steep learning curve associated with an ATE. To provide the same types of instrumentation as the ATE, each platform is outfitted with a personal computer, a National Instruments' multifunction data acquisition card, a general-purpose interface bus (GPIB) card, and a traditional set of analog electronics bench equipment (oscilloscope, function generator, power supply, multimeter).

The multifunction data acquisition card provides signal digitization capability and static digital I/O. Ten of the stations use a 12-bit PCI 6025E data acquisition card and one station uses a higher resolution 16-bit PCI 6035E card. The GPIB card gives the system control over the bench equipment. Each station is also equipped with National Instruments' LabVIEW to allow the PC to communicate with the hardware and to post-process data. National Instrument's LabVIEW graphical programming language allows the students to easily develop automated tests. The obvious differences between the PC-based platforms and the ATE are cost, speed, resolution, and sensitivity.

\section{Testing Procedure}

To acquire data for correlation purposes, the class is broken into teams of two or three. Each team then has to use both platforms to test the static linearity of an 8-bit DAC (DAC0808, National Semiconductor, Inc.). This allows each team to correlate test results between the two platforms and allows inter-team correlation of results as well. Linearity testing consists of acquiring the output voltage of the DAC for all possible input codes and then calculating the six major static characteristics of the DAC including gain, gain error, offset, offset error, integral non-linearity (INL), and differential nonlinearity (DNL). [2] An example of this data can be seen in Figure 2.

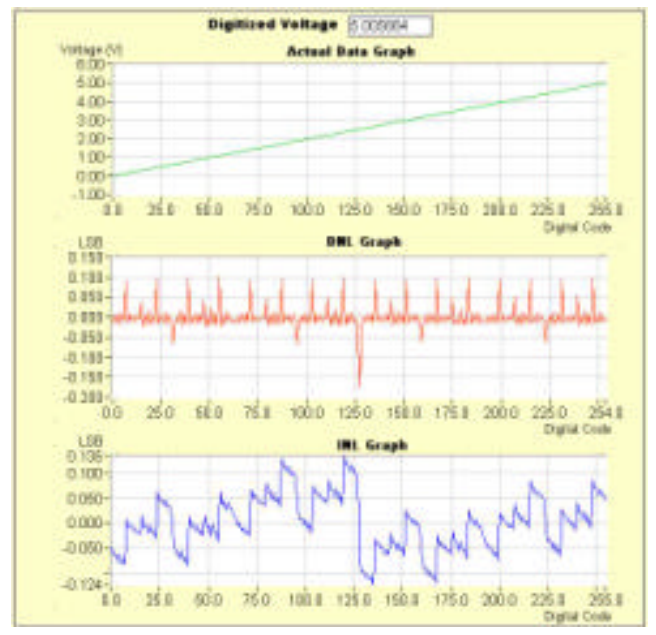

Figure 2 - Example of data from an 8-bit DAC including DNL and INL test parameters.

Proceedings of the 2002 American Society for Engineering Education Annual Conference \& Exposition Copyright (O2002, American Society for Engineering Education 
Gain and offset are single value parameters that characterize a DAC's overall input code to ouput voltage relationship (They are the calculated slope and intercept of the raw data as seen in the top graph of Figure 2). DNL and INL are different in that they characterize errors that occur at each individual input code value. DNL shows the difference in uniformity in the step sizes between adjacent DAC codes. INL shows the deviation of the DAC's output from the ideal output at each input code value. Thus they are a set of points rather than a single value.

Before the devices can be tested, they have to be interfaced to each of the two platforms. This includes building any device support circuitry as well as device-to-tester connections. For this experiment, it is critical that the device being tested requires some form of external support circuitry. This ensures that each team will have varied results and leaves the possibility for error that will show up when teams compare results. For the DAC0808, external support circuitry includes current setting resistors and an output current-to-voltage converter circuit. The test circuit used can be seen in Figure 3.

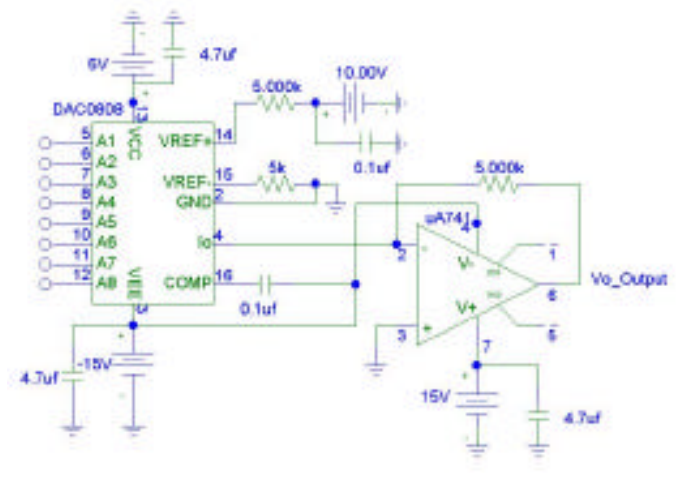

Figure 3 - Device interface schematic.

To build the interface, each team is given one of the Teradyne prototyping interface boards mentioned previously. All external support circuitry for the DAC as well as tester connections are built on this board. To ensure test uniformity within individual student teams, the same interface circuitry was used on both test platforms. A picture of a populated interface board can be seen in Figure 4.

Next, the teams have to write and perform the required tests on the two platforms. The programs to run the tests for the DAC0808 on the A567 are written in IMAGE. The program first sets up the necessary source voltages. Then, a digital pattern generator is used to provide the input codes and the output of the DAC is measured with a DC voltmeter. The voltmeter has a $20 \mathrm{~V}$ range and 14-bits of resolution. The test program is complex enough to leave the possibility of error that can show up during measurement correlation. 


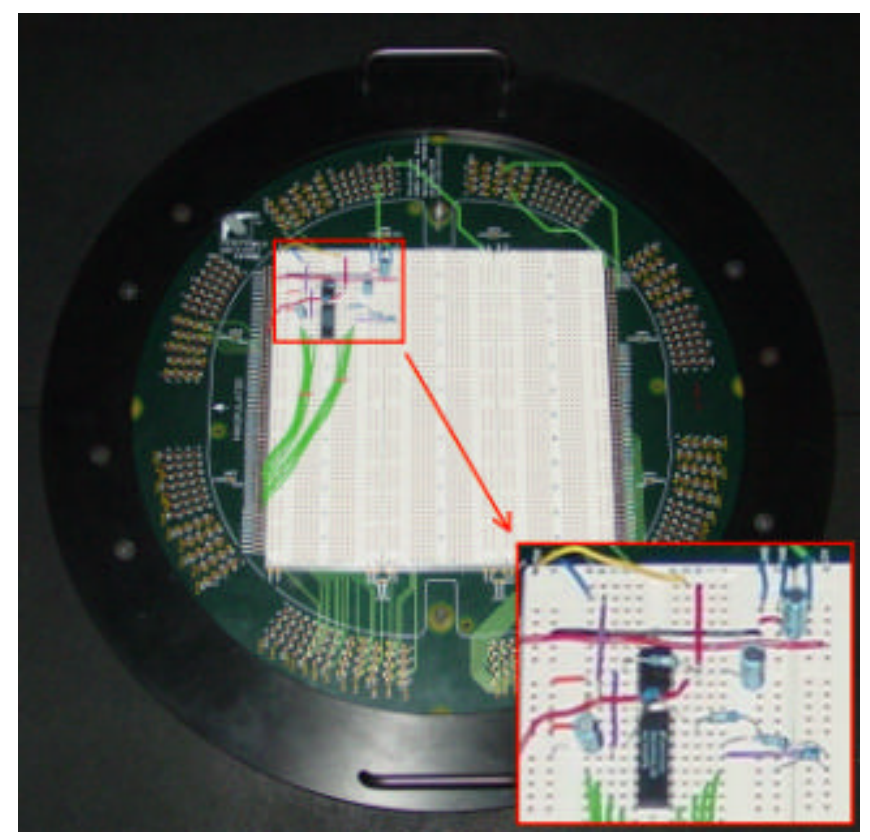

Figure 4 - Device interface board with including the device to be test and external circuitry.

To replicate the test on the PC-based platform, the students use two Agilent E3631A triple output power supplies to supply the voltages required by the DAC. The multifunction data acquisition card is used to provide the eight static digital I/O lines needed for the input. Either the 12-bit PCI 6025E or the 16-bit PCI 6035E is used to digitize the output voltage of the DAC. This means that during data correlation, the students can see the effect of using up to three different measurement devices.

LabVIEW code is then written to measure the output voltage at each of the 256 input codes and calculate the desired parameters. Finally, the students are required to repeat the tests multiple times on each platform to provide statistical information.

\section{Examples of Correlation Results}

Once results have been collected from the two platforms, students can correlate the data in two ways. First, each team can correlate their own test data between the two test platforms. This allows the students to see the effects of using different test equipment. Because the differences between the digital test instruments are insignificant due to the static nature of these tests, the effects of using different instruments to digitize the DAC output voltage are isolated and easy to identify. Second, teams can correlate their data to that obtained from other teams on the same platform, demonstrating the differences caused by using different test code and hardware setups. This typically allows groups to identify errors they may have made in their test program and hardware setup. The following presents some examples of correlation results that students are likely to see. 


\section{Comparison of Data from Different Test Platforms}

In this example, test data is compared between three measurement platforms mentioned previously (typically the students only do two). These include the Teradyne platform using a high precision 14-bit voltmeter and the PC-based test platform using either the 12-bit or 16-bit digitizer. On each platform, the tests are repeated twenty separate times and each time all of the test parameters are calculated. The results are then averaged and the standard deviations are computed. This statistical data yields information about repeatability and noise.

Table 1 shows a comparison of the gain and offset parameters. The first thing that can be seen is that the results from each of the test platforms agree fairly well. However, on closer inspection one can notice that the 12-bit results are slightly different than those from the other two platforms. While this could imply that the 12-bit results are not as accurate as the results from the Teradyne and the 16-bit platform, there is not enough data to be conclusive. However, looking at the results from the all codes test gives us additional information.

Table 1 - Gain and offset results from the three test platforms.

\begin{tabular}{|l|l|l|l|l|l|}
\hline Test & Ideal Gain & Actual Gain & Std. Dev. & Gain Error & Std. Dev. \\
\hline \hline 12-Bit PC-Based & 0.038906 & 0.039062 & 0.000000 & -0.400999 & 0.000672 \\
\hline 16-Bit PC-Based & 0.038906 & 0.039084 & 0.000000 & -0.456436 & 0.000247 \\
\hline Teradyne & 0.038906 & 0.039082 & 0.000000 & -0.450837 & 0.000791 \\
\hline
\end{tabular}

\begin{tabular}{|l|l|l|l|l|l|}
\hline Test & Ideal Offset & Actual Offset & Std. Dev. & Offset Error & Std. Dev. \\
\hline \hline 12-Bit PC-Based & 0.000000 & -0.002953 & 0.000025 & -0.002953 & 0.000025 \\
\hline 16-Bit PC-Based & 0.000000 & -0.003457 & 0.000019 & -0.003457 & 0.000019 \\
\hline Teradyne & 0.000000 & -0.003618 & 0.000016 & -0.003618 & 0.000016 \\
\hline
\end{tabular}

Figures 5 through 7 show the raw data, the DNL results, and the INL results respectively. Again, the three plots show relatively good correlation in the overall data between test platforms. The average plots of the raw data, the DNL and the INL all show similar trends. One can see that the 12-bit digitizer results for DNL and INL are missing the detail seen in the other two plots. In fact, the 12-bit plot shows "dead-spots" especially in the DNL plot. Students will typically arrive at the correct conclusion that the 12-bit device, being only sixteen times more accurate than the device being tested, does not have enough resolution to show the same detail as the Teradyne.

Finally, the standard deviation plots can be studied to gain an understanding of the noise, or repeatability, of the measurement. One can see that the noise from the 12-bit digitizer is the largest with the noise from the 16-bit digitizer being the smallest. Because the noise in the 16-bit digitizer measurements is not as small as expected, these results also lend themselves well to a discussion of effective number of bits and to test equipment noise versus intrinsic circuit noise. 


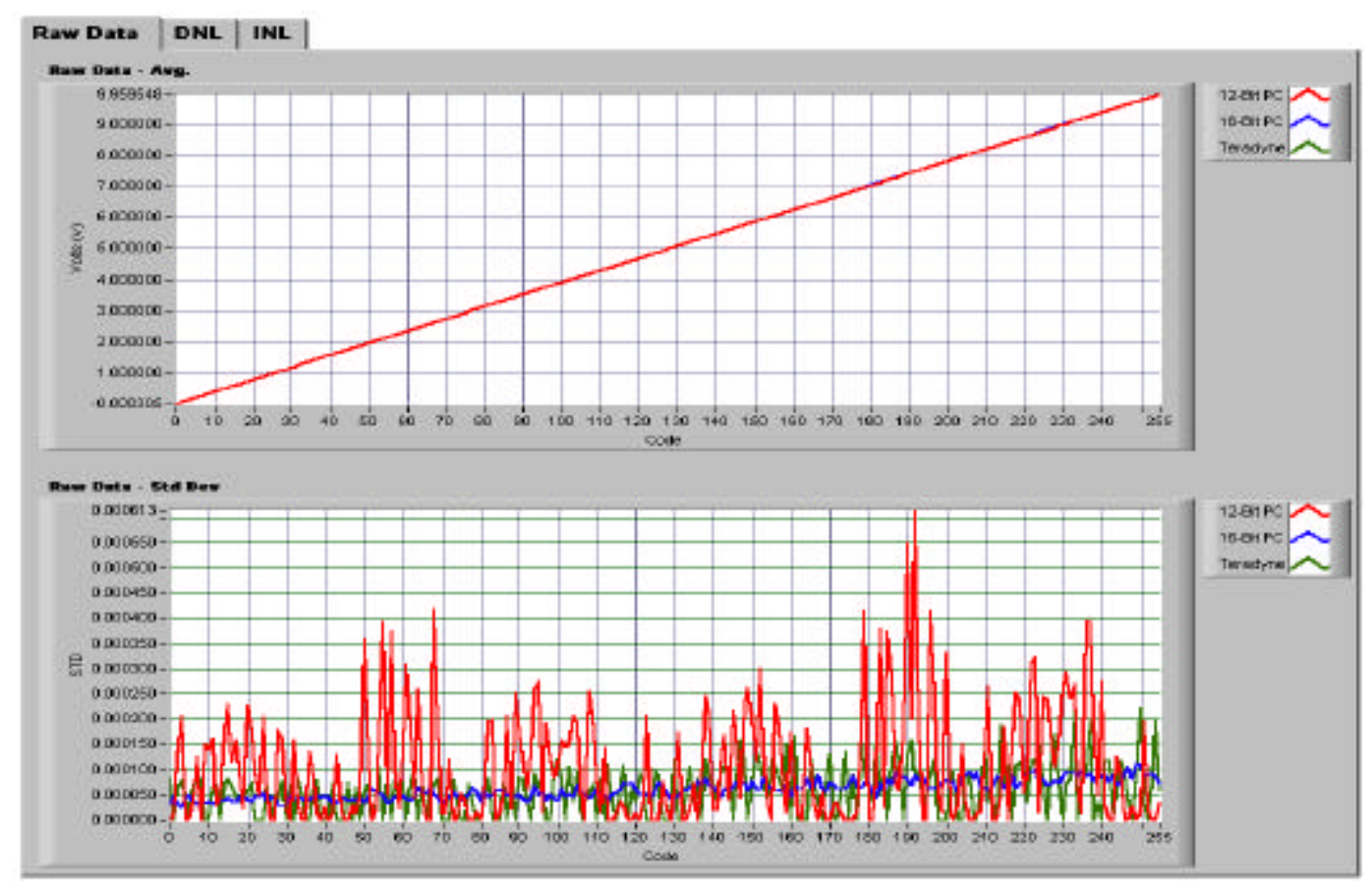

Figure 5 - Raw data taken from each system plotted against each other (top) with the standard deviation of each setup (bottom).

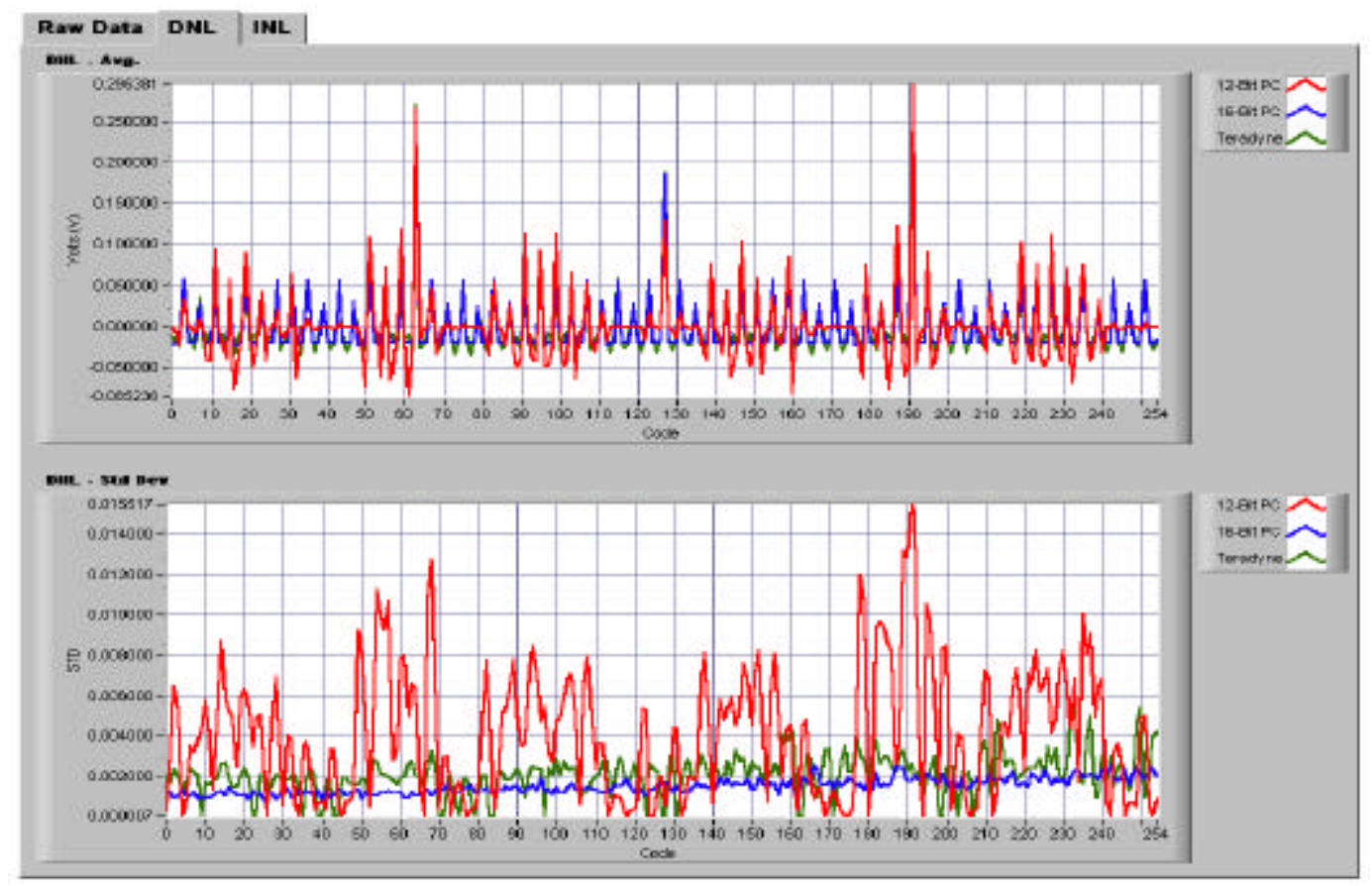

Figure 6 - Differential non-linearity plot (top) and the DNL standard deviation plot (bottom).

Proceedings of the 2002 American Society for Engineering Education Annual Conference \& Exposition Copyright (C2002, American Society for Engineering Education 


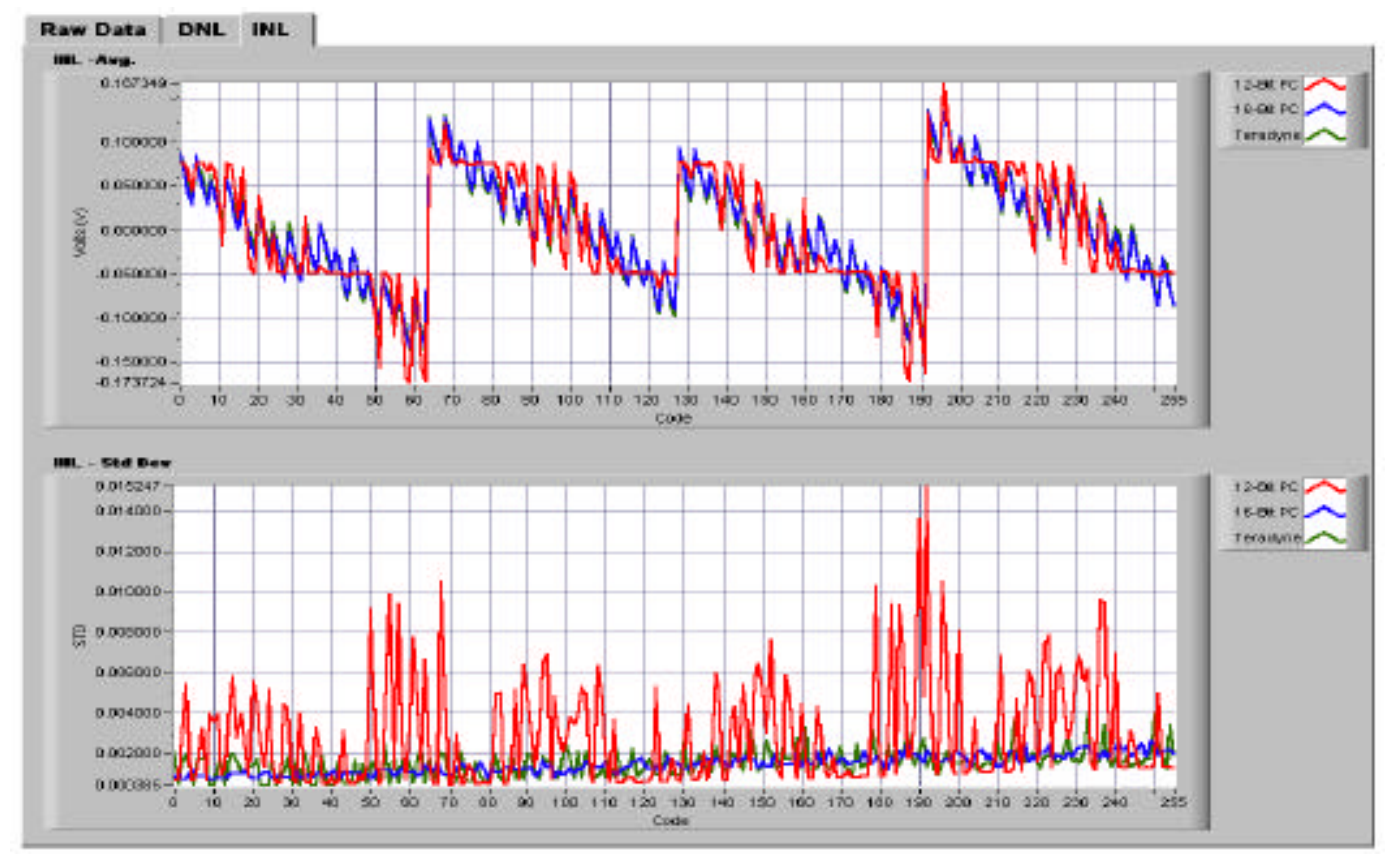

Figure 7 - Integral non-linearity plot (top) and the INL standard deviation plot (bottom).

A second problem was found while correlating data from different platforms. During the initial setup on the Teradyne system, two separate $-15 \mathrm{~V}$ power supplies were used to power different portions of the circuit. On the PC-based platform, a single $-15 \mathrm{~V}$ power supply had been used. Upon correlating the data sets from both platforms, the Teradyne showed excessive noise in the DNL plots near one of the major carrier changes. In production, excessive noise can lead to good devices being failed during the test process. This problem was traced to the use of the separate power supplies. Upon changing the setup to use a single supply, the excessive noise disappeared.

\section{Comparison of Data from Teams Using Different Interface Circuitry}

This example shows how correlation can point out differences between tests done using different interface designs. Often, students given the same circuit to build, will take liberties with component values. For the DAC0808, the current to voltage converter requires a precision $5 \mathrm{KOhm}$ resistor. Figure 8 shows the raw data acquired using different resistor values. Specifically, one can see a major difference between the data acquired using a precision $5 \mathrm{KOhm}$ resistor in the output circuit versus the data acquired using a $4.7 \mathrm{KOhm}$ resistor that would be available in a student's parts kit. Upon noticing such an anomaly, the student teams would then have to troubleshoot the cause. The major change in gain would most likely lead them to the gain resistor in the feedback of the op amp. Giving students this type of real world experience helps reiterate the importance of communication, correlation, and basic troubleshooting skills. 


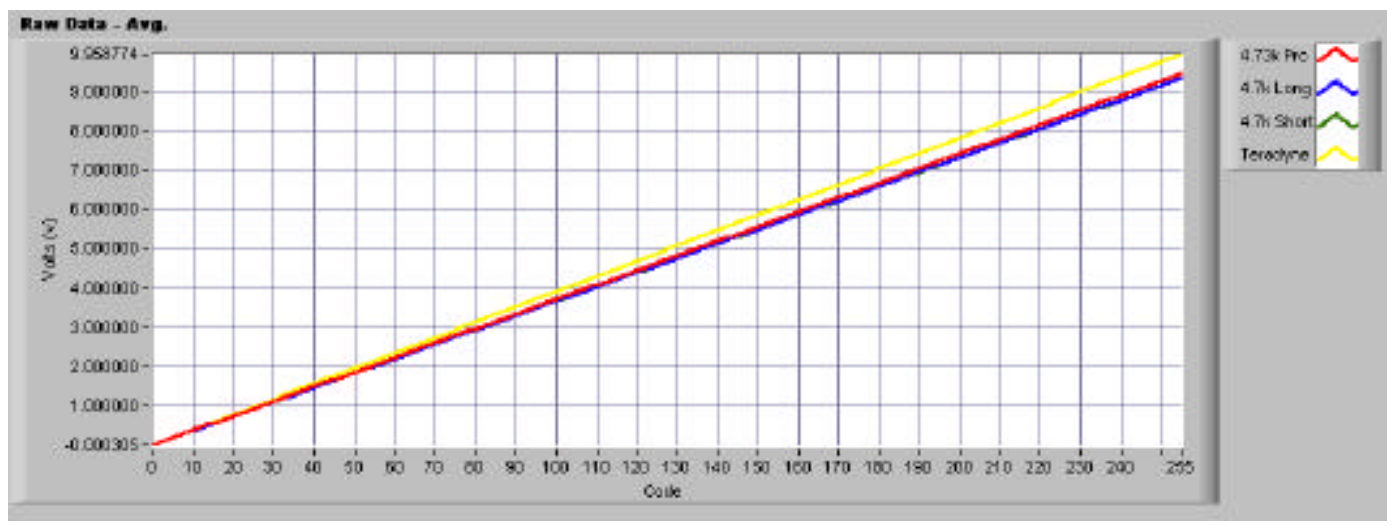

Figure 8 - Results from a test in which a different sized resistor was used in the feedback for the op amp. Long and short refer to the length of the leads of the resistor.

\section{Comparison of Data from Teams Using Different Test Software}

As with hardware setups many test setups also include software issues that lead to non-correlating results. Different test platforms may use different programming and software packages. Having to create separate programs on different platforms can lead to a multitude of issues; some easily noticeable and others that might consume a large number of man-hours to troubleshoot. It is very important that the programs on all platforms collect the data in the same manner and process it using similar algorithms. It is common practice in industry to have multiple teams testing the same design using different test platforms. Helping to identify processing algorithm errors that might not show up in the results until they are compared with results from a different platform. The ability to test the validity of a data set against other data sets can help sidestep major problems later on in the production phase of a design's life cycle.

In the case of the characterization of the DAC0808, separate students performed the initial tests. It was not until the data was reviewed together that a second anomaly in noise levels, prior to the power supply issue, was noticed. To determine the difference in noise levels, the students turned to their code to ensure both setups were acquiring the data in similar fashions. It was found that the Teradyne test code was only averaging five samples per code, where as the PC-Based system was averaging 100 samples per code. Such errors can be contributed to the lack of communication between groups testing the same device. By correlating data between test platforms, a level of quality control is obtained.

\section{Conclusions}

Through the Semiconductor Test Initiative between industry and the EET program at Texas A\&M, 
students are learning the importance of using the correlation of data acquired from multiple test platforms to aid in mixed-signal device testing. By using two different mixed-signal test platforms to test the same device, they are finding that they can successfully use a comparison of results to debug interface hardware, debug test software and to understand the effects of using different measurement instrumentation. Since the principals discussed here are applicable to any type of engineering, the lessons learned will prepare students for engineering careers, especially in mixed signal semiconductor testing.

Bibliography

1. Fink, R. and Porter, J. "Mixed-Signal Test Initiative in Engineering Technology," Proc. of the 1999 Gulf Southwest ASEE Conference, Dallas, TX (March, 1999).

2. Burns, M. and Roberts, G. An Introduction to Mixed-Signal IC Test and Measurement. (New York: Oxford University Press, 2001).

\section{MICHAEL WARREN}

Michael Warren graduated in December 2000 with the BS in Engineering Technology. He is currently working on his MS in the Information and Operations Management Department at Texas A\&M University. He also works as a graduate research assistant in the Virtual Instrumentation Laboratory in the Department of Engineering Technology and Industrial Distribution.

\section{DANA BURNETT}

Dana Burnett is pursuing a BS in Engineering Technology at Texas A\&M University. She also works as an undergraduate research assistant in the Texas Instruments Mixed Signal Test Laboratory in the Department of Engineering Technology and Industrial Distribution.

\section{JAY PORTER}

Jay R. Porter joined the Department of Engineering Technology and Industrial Distribution at Texas A\&M University in 1998 as an Assistant Professor and currently works in the areas of mixed-signal circuit testing and virtual instrumentation development. He received the BS degree in electrical engineering (1987), the MS degree in physics (1989), and the Ph.D. in electrical engineering (1993) from Texas A\&M University.

\section{RAINER FINK}

Rainer Fink received the BS degree in biomedical engineering (1988), the MS degree in biomedical engineering (1992), and the Ph.D. in biomedical engineering (1995) from Texas A\&M University. After finishing his Ph.D., he was a lecturer in the Bioengineering Program and the Department of Engineering Technology at Texas A\&M University. In August 1996, he joined the Electronics Engineering Technology faculty at Texas A\&M University. His research activities include mixed-signal testing, analog circuit design and biomedical electronics.

Proceedings of the 2002 American Society for Engineering Education Annual Conference \& Exposition Copyright (O2002, American Society for Engineering Education 